\title{
„Ich habe den Leistungssport geliebt und gelebt“
}

\section{Felicitas Merker}

Felicitas Merker ist eine ehemalige deutsche Gehörlosensportlerin. Im Siebenkampf holte sie unter anderem bei den Deaflympics 2017 die Bronzemedaille oder Silber-bzw. Bronzemedaillen (2015 und 2012) bei Europameisterschaften. Als Auszeichnung wurde ihr daraufhin das Silberne Lorbeerblatt verliehen.

Der Leistungssport war lange ein sehr wichtiger Bestandteil meines Lebens. Er hat mir viel gegeben, wofür ich immer dankbar sein werde. Ich durfte mein Land zweimal bei den Deaflympics, den vom IOC anerkannten Weltspielen der Gehörlosen, repräsentieren und konnte dabei einmal sogar die Bronzemedaille gewinnen. Nach meinem Karriereende bin ich dem Sport treu geblieben und versuche nun, einen Teil meiner Leidenschaft und positiven Erfahrungen weiterzugeben.

Begonnen hat alles ganz klassisch: Nach einer sehr erfolgreichen Teilnahme bei Bundesjugendspielen meldeten mich meine Eltern im Leichtathletikverein an. Das war ein ganz regulärer Verein ohne spezielle Angebote für Menschen mit einer Hörbehinderung, aber die Trainingsgruppe und meine Trainer*innen nahmen mich damals gleich toll auf.

Gerade in der Schulzeit war der Sport für mich immer ein wichtiger Anker. Ich war zwar eine sehr gute Schülerin und wurde auch von meinen Lehrer*innen aufgrund meiner Beeinträchtigung immer besonders gefördert. In der Klasse war aber gerade diese Vorzugsbehandlung auch der Grund für Neid und mitunter sogar für eine Form von Mobbing. Und obwohl mir das Lernen leichtfiel, war doch der Geräuschpegel in der Klasse immer eine große Herausforderung für meine Konzentration. Der Unterricht war daher oft sehr anstrengend für mich. Sobald ich aber nachmittags auf dem Sportplatz war und mich auspowern konnte, war das vergessen. Der Sport hat mir schon damals viel Energie gegeben.

Die Begeisterung für den Sport hat mich bis zu den Deaflympics gebracht. Ähnlich wie die Olympischen Spiele und die Paralympics zeichnen sich auch unsere Weltspiele durch ein ganz besonderes Flair aus. Wenn die verschiedenen Nationalitäten mit ihren unterschiedlichen Gebärdensprachen aufeinandertreffen, entsteht eine besondere Stimmung.

Menschen mit einer Hörbehinderung haben sehr spezielle Bedürfnisse, weswegen ich auch einer Aufnahme in das Programm der Paralympics 
skeptisch gegenüberstehe. Das würde zwar auch einige Vorteile wie eine stärkere mediale Aufmerksamkeit nach sich ziehen, ich hätte aber die Befürchtung, dass ein Teil unserer besonderen Kultur verloren ginge.

Leider ist es bei den Deaflympics nicht üblich, dass die Athlet*innen in einem eigenen Komplex wie etwa dem Olympischen Dorf zusammenwohnen. Da der Austragungsort nicht wie der der Paralympics an die Olympischen Spiele gekoppelt ist, können wir auch keine bestehenden Anlagen nutzen. So beschränkt sich der intensive Austausch mit anderen Nationen auf die gemeinsamen Eröffnungs- und Schlussfeiern.

Auch auf der persönlichen Ebene hat mich der Sport sehr geprägt. Werte wie der Respekt vor der Leistung anderer oder Fair Play, die mir in Fleisch und Blut übergegangen sind, versuche ich, auch in allen anderen Bereichen meines Lebens umzusetzen. Meine Disziplin, der Siebenkampf, ist sogar ein Stück weit Teil meines Charakters geworden. Vergangene Leistungen abhaken, sich neu fokussieren und den Blick auf die nächsten anstehenden Aufgaben zu richten ist auch im beruflichen Kontext eine wichtige Ressource. Ich habe über meine Erfolge im Sport ein großes Maß an Selbstbewusstsein gewonnen, das für mich als junge Frau mit einem beeinträchtigten Hörvermögen sonst vielleicht gefehlt hätte. Und über die Auseinandersetzung mit den eigenen Bedürfnissen sowie leistungshemmenden und -fördernden Faktoren habe ich auch einen sehr verantwortungsvollen Umgang mit mir selbst entwickeln können.

Das Gefühl, sich in harten Trainingseinheiten zu quälen und die Zufriedenheit, mit der man anschließend ins Bett geht, fehlt mir nach meinem Karriereende 2019 manchmal. Auch das Adrenalin und die Nervosität vermisse ich ab und zu. Klar ist aber auch: Als Leistungssportlerin habe ich dem sportlichen Erfolg viel untergeordnet. Die Freiheit, nach Lust und Laune Sport zu treiben oder auch einmal die Beine hochzulegen, neue Sportarten ohne Angst vor Verletzungen auszuprobieren oder spontan in den Urlaub zu fahren, ist eine neue Lebensqualität, die ich nicht missen möchte.

Meine Erfahrungen als Leistungssportlerin im Gehörlosensport möchte ich weitergeben. Nach dem Abitur habe ich daher auch Sport studiert und begleite nun als Referentin für Leistungssport unsere Athlet*innen im Deutschen Gehörlosen-Sportverband. Neben meiner Rolle als Ansprechpartnerin in allen Fragen der sportlichen Karriere möchte ich aber auch helfen, den gesamten Gehörlosensport voran zu bringen. Mein Traum ist es, dass die Deaflympics irgendwann ein ähnliches Standing genießen wie die Paralympics. Das ist ein ambitioniertes Ziel, aber der Fokus auf neue Ziele und große Herausforderungen bereiten einer Siebenkämpferin wenig Probleme. 